\title{
ASYMPTOTIC BEHAVIOR FOR A COALESCENCE PROBLEM
}

\author{
OSCAR BRUNO, AVNER FRIEDMAN, AND FERNANDO REITICH
}

\begin{abstract}
Consider spherical particles of volume $x$ having paint on a fraction $y$ of their surface area. The particles are assumed to be homogeneously distributed at each time $t$, so that one can introduce the density number $n(x, y, t)$. When collision between two particles occurs, the particles will coalesce if and only if they happen to touch each other, at impact, at points which do not belong to the painted portions of their surfaces. Introducing a dynamics for this model, we study the evolution of $n(x, y, t)$ and, in particular, the asymptotic behavior of the mass $x n(x, y, t) d x$ as $t \rightarrow \infty$.
\end{abstract}

\section{INTRODUCTION}

Aerosol dynamics is often modelled by an evolution equation for the number density $n(x, t)$ of the particles of volume $x$,

$$
\begin{aligned}
\frac{\partial n(x, t)}{\partial t}= & -n(x, t) \int_{0}^{\infty} \varphi(x, \xi) n(\xi, t) d \xi \\
& +\frac{1}{2} \int_{0}^{x} \varphi(x-\xi, \xi) n(x-\xi, t) n(\xi, t) d \xi
\end{aligned}
$$

where $\varphi(x, \xi)$ is the collision rate between particles $x$ and $\xi$. The underlying assumptions are that particles of any volume $x$ are homogeneously distributed at each time $t$, and that when two particles of volumes $x$ and $\xi$ collide, they coalesce, thus forming a new particle of size $x+\xi$. The right-hand side in (0.1) is called the coagulation operator. The choice of $\varphi$ depends on the physical environment of the particles (see [2, 5]). Some models incorporate also a fragmentation operator, and some models study the discrete version of (0.1) whereby

$$
n(x, t)=\sum_{j=1}^{\infty} n_{j}(t) \delta(x-j) \quad(\delta=\text { Dirac function })
$$

For existence and uniqueness theorems, and numerical calculations, we refer to [1-12 and 14, 15].

Recently David Ross and Tom Whitesides [13] introduced a new coagulation model. They consider spherical particles (such as oil drops) that are partially covered with surfactant (or "paint"). Collision between two particles will result

Received by the editors March 7, 1991.

1991 Mathematics Subject Classification. Primary 35Q20, 35B40.

Key words and phrases. Particles, collision, coalescence, asymptotic behavior. 
in coalescence if and only if the particles touch each other on their "unpainted" surface at the moment of collision. If we denote by $y$ the fraction of surface area of the particle which is painted, then the number density $n(x, y, t)$ of particles $(x, y)$ satisfies the evolution equation given in (1.1) below.

Several questions arise

(a) What is the effect of the surfactant for large times? Do all particles become painted as $t \rightarrow \infty$ ?

(b) Does the asymptotic distribution of the mass depend continuously on the initial distribution?

In this paper we answer these questions. Regarding (a) we prove that

$$
\int_{0}^{1} \int_{0}^{\infty} x n(x, y, t) d x d y \rightarrow 0 \text { if } t \rightarrow \infty,
$$

i.e., all the mass is contained in completely painted particles as $t \rightarrow \infty$. To do this we shall introduce in $\S 4$ a more detailed description of the evolution of the system than the one given by (1.1).

Denoting the limiting density number by $n(x, y, \infty)$, we also prove that

$$
\iint_{P}(1+x)|n(x, y, t)-n(x, y, \infty)| d x d y \rightarrow 0 \text { if } t \rightarrow \infty,
$$

where $P$ denotes the space of all particles $(x, y)$ (when two particles coalesce they may produce a particle $(x, y)$ with $y>1$; however, $\left.y \leq 2^{1 / 3}\right)$.

Take a family of initial conditions $n_{\lambda}(x, y) \quad(0 \leq \lambda \leq 1)$ and denote by $n_{\lambda}(x, y, t)$ the solution corresponding to $n_{\lambda}(x, y, 0)=n_{\lambda}(x, y)$. We shall prove the following stability result which answers positively question (b) above:

$$
\begin{aligned}
& \text { if } \iint_{P}(1+x)\left|n_{\lambda}(x, y)-n_{0}(x, y)\right| d x d y \quad \text { as } \lambda \rightarrow 0 \\
& \text { then } \iint_{P}(1+x)\left|n_{\lambda}(x, y, \infty)-n_{0}(x, y, \infty)\right| d x d y \rightarrow 0 \text { as } \lambda \rightarrow 0 .
\end{aligned}
$$

The above results are first proved for the case where the initial data are continuous functions. In the last section of this paper we extend the results to initial data that are measures, thus enabling one to compute an approximation for the mass distribution $x n(x, y, \infty) d x$ by working with solutions of the form

$$
n_{\lambda}(x, y, t)=\sum_{m, k} R_{m k}(t) \delta\left(x-x_{m}\right) \delta\left(y-y_{k}\right) \quad(\text { finite sum })
$$

whereby $R_{m k}(t)$ are solutions to systems of ordinary differential equations. A different numerical approach based on Monte Carlo simulations was carried out by D. Ross and T. Whitesides [13].

\section{THE MODEL}

Spherical particles with variable volume $x$ are distributed in the entire space $\mathbb{R}^{3}$. The surface of each particle is partially covered by surfactant; we refer to the surfactant as "paint" and to the covered area as the "painted area". We denote by $y$ the fraction of the painted surface area of the spherical particle, i.e., the total painted area is $\gamma y x^{2 / 3}$ where $\gamma=4 \pi /\left(\frac{4 \pi}{3}\right)^{2 / 3}$; for simplicity we drop the factor $\gamma$, since it will cancel out in all subsequent calculations. 
We characterize each particle by $(x, y)$. The model assumes that at each time $t$ the particles are distributed homogeneously. We denote by $n(x, y, t)$ the density number of particles $(x, y)$, that is, the number of particles in $(x, x+\Delta x) \times(y, y+\Delta y)$ is approximately $n(x, y, t) \Delta x \Delta y$.

Denote by $\varphi(x, \xi)$ the rate at which particles with volumes $x$ and $\xi$ will collide, per unit time. The model assumes that when two particles $(x, y)$ and $(\xi, \eta)$ collide, they coalesce if and only if they touch each other at the unpainted portion of their surface. More precisely, the number of aggregations of particles in $(x, x+\Delta x) \times(y, y+\Delta y)$ and $(\xi, \xi+\Delta \xi) \times(\eta, \eta+\Delta \eta)$ per unit time is proportional to the numbers $n(x, y) \Delta x \Delta y, n(\xi, \eta) \Delta \xi \Delta \eta$, to the fractions of surface areas $1-y$ and $1-\eta$ that are unpainted, and to the collision rate $\varphi(x, \xi)$. This assumption leads to the following evolution equation

$$
\begin{aligned}
\frac{\partial n(x, y, t)}{\partial t}= & -\int_{0}^{1} \int_{0}^{\infty} \varphi(x, \xi)(1-\eta)(1-y)^{*} n(\xi, \eta, t) n(x, y, t) d \xi d \eta \\
+ & \frac{1}{2} \int_{0}^{1} \int_{0}^{x} \varphi(\xi, x-\xi)(1-\eta)\left(1-\frac{y x^{2 / 3}-\eta \xi^{2 / 3}}{(x-\xi)^{2 / 3}}\right)^{*} \\
& \times \eta(\xi, \eta, t) n\left(x-\xi, \frac{y x^{2 / 3}-\eta \xi^{2 / 3}}{(x-\xi)^{2 / 3}}, t\right) \frac{x^{2 / 3}}{(x-\xi)^{2 / 3}} d \xi d \eta
\end{aligned}
$$

where

$$
(1-s)^{*}= \begin{cases}0 & \text { if } s>1 \text { or if } s<0, \\ 1-s & \text { if } 0<s<1,\end{cases}
$$

the last notation means that the last integral

$$
\int_{0}^{x} \int_{0}^{1} \cdots d \eta d \xi \text { is actually } \int_{0}^{x} \int_{\max \left(0, y\left(\frac{x}{\xi}\right)^{2 / 3}-\left(\frac{x-\xi}{\xi}\right)^{2 / 3}\right)}^{\min \left(1, y\left(\frac{x}{\xi}\right)^{2 / 3}\right)} \cdots d \eta d \xi .
$$

To explain the somewhat complicated form of the integrand in the last integral in $(1.1)$, we observe that if a particle $(x, y)$ collides with a particle $(\xi, \eta)$ so that they coalesce, then the new particle $(z, s)$ is given by

$$
z=x+\xi, \quad s z^{2 / 3}=y x^{2 / 3}+\eta \xi^{2 / 3} .
$$

We also write

$$
(z, s)=(x, y) \oplus(\xi, \eta)
$$

and call $(z, s)$ the impact of $(x, y)$ and $(\xi, \eta)$. Thus except for the factor $x^{2 / 3} /(x-\xi)^{2 / 3}$, the integrand in the last integral in (1.1) represents all particles $(\xi, \eta),(x-\xi, \tilde{s})$ whose impact is $(x, y)$; the expression

$$
\left(y x^{2 / 3}-\eta \xi^{2 / 3}\right) /(x-\xi)^{2 / 3}
$$

for $\tilde{s}$ follows from the definition (1.2). The factor $\frac{1}{2}$ in front of the integral accounts for the fact that collisions were counted twice in the integral. To explain the factor $x^{2 / 3} /(x-\xi)^{2 / 3}$, consider all the particles in $(x, x+\Delta x) \times$ $(y, y+\Delta y)$ obtained by impact of $(\xi, \eta)$ and particles from $(p, p+\Delta p) \times$ $(q, q+\Delta q)$. Then

$$
x=p+\xi, \quad y x^{2 / 3}=q p^{2 / 3}+\eta \xi^{2 / 3},
$$


and

$$
x+\Delta x=p+\Delta p+\xi, \quad(y+\Delta y) x^{2 / 3}=(q+\Delta q) p^{2 / 3}+\eta \xi^{2 / 3} .
$$

It follows that

$$
\frac{\Delta p \Delta q}{\Delta x \Delta y}=\frac{x^{2 / 3}}{(x-\xi)^{2 / 3}}
$$

so that, with $p=x-\xi, q=\left(y x^{2 / 3}-\eta \xi^{2 / 3}\right) /(x-\xi)^{2 / 3}$ we need to put, in the last integral in (1.1),

$$
n(\xi, \eta, t) n(p, q, t) x^{2 / 3} /(x-\xi)^{2 / 3} \quad(\text { rather than } n(\xi, \eta, t) n(p, q, t)) .
$$

The impact of two particles may result in a particle $(x, y)$ with $y>1$; however, $y \leq 2^{1 / 3}$. For this reason the particle space is taken to be

$$
P=\left\{(x, y) ; 0<x<\infty, 0 \leq y \leq 2^{1 / 3}\right\} .
$$

Throughout this paper we assume that

$$
\begin{gathered}
\varphi(x, \xi)=\varphi(\xi, x), \quad \varphi \text { is continuous }, \\
0 \leq \varphi \leq C<\infty \quad(C \text { constant }), \text { and } \\
\varphi(x, \xi)>0 \quad \text { if } x>0, \xi>0
\end{gathered}
$$

We shall impose an initial condition

$$
n(x, y, 0)=n_{0}(x, y)
$$

and assume that

$$
\begin{array}{ccc}
n_{0}(x, y) & \text { is continuous, } \\
n_{0}(x, y) \geq 0, & n_{0}(x, y)=0 & \text { if } y \geq 1, \\
\iint_{P} n_{0} d x d y<\infty, & \iint_{P} x n_{0}(x, y) d x d y<\infty .
\end{array}
$$

Theorem 1.1. Under the assumptions (1.3), (1.5), there exists a unique solution $n(x, y, t)$ of $(1.1),(1.4)$ such that $n(x, y, t) \geq 0$ and, for every $T>0$,

$$
\iint_{P} n(x, y, t) d x d y \leq C_{T}, \quad \iint_{P} x n(x, y, t) d x d y \leq C_{T}
$$

if $0 \leq t \leq T$; furthermore, $n(x, y, t)$ is continuous.

The proof follows by minor changes from Melzak [11]. This proof is briefly outlined in $\S 5$, where the theorem is generalized to measure-valued solutions. As in [11] we also find that $n(x, y, t)$ is analytic in $t$ with values in $L^{1}(P)$. The uniqueness assertion holds also if $n$ is not assumed to be nonnegative.

Remark 1.1. The last assumption in (1.3) is not needed for Theorem 1.1 or for all other results proved in this section; it will be needed however in the subsequent sections.

Remark 1.2. The boundedness of $\varphi$ is needed only for uniqueness. Indeed, the method of Friedman and Reitich [3] (who considered a more general evolution equation than $(0.1))$ can be modified to prove existence provided $\varphi(x, \xi) \leq$ $\sigma(x+\xi)$ where $\sigma(r) / r \rightarrow 0$ as $r \rightarrow \infty$.

The next theorem establishes conservation of mass and paint. 
Theorem 1.2. The following relations hold:

$$
\begin{aligned}
\iint_{P} x n(x, y, t) d x d y & =\iint_{P} x n_{0}(x, y) d x d y, \\
\iint_{P} y x^{2 / 3} n(x, y, t) d x d y & =\iint_{P} y x^{2 / 3} n_{0}(x, y) d x d y .
\end{aligned}
$$

Proof. Multiplying (1.1) by $x$ and integrating over $P$ we get

$$
\begin{aligned}
& \frac{\partial}{\partial t} \iint_{P} x n=-\iint_{P} \iint_{P} x \varphi(x, \xi)(1-\eta)^{*}(1-y)^{*} n(\xi, \eta, t) n(x, y, t) \\
& \quad+\frac{1}{2} \iint_{P} d y d x \int_{0}^{1} d \eta \int_{0}^{x} d \xi x \varphi(\xi, x-\xi)(1-\eta)^{*} \\
& \quad \times\left(1-\frac{y x^{2 / 3}-\eta \xi^{2 / 3}}{(x-\xi)^{2 / 3}}\right)^{*} n(\xi, \eta, t) \\
& \quad \times n\left(x-\xi, \frac{y x^{2 / 3}-\eta \xi^{2 / 3}}{(x-\xi)^{2 / 3}}, t\right) \frac{x^{2 / 3}}{(x-\xi)^{2 / 3}} .
\end{aligned}
$$

In the last integral we change the order of integration of $(x, y)$ and $(\xi, \eta)$ and then change variables,

$$
\begin{aligned}
& z=x-\xi \quad(\xi \text { fixed, so that } 0<z<\infty), \\
& s=\frac{y x^{2 / 3}-\eta \xi^{2 / 3}}{z^{2 / 3}}(\xi, \eta \text { fixed }) .
\end{aligned}
$$

Noting that

$$
d z d s=\frac{x^{2 / 3}}{(x-\xi)^{2 / 3}} d x d y
$$

we get

$$
\begin{aligned}
& \frac{1}{2} \int_{0}^{1} d \eta \int_{0}^{\infty} d \xi \int_{0}^{2^{1 / 3}} d s \int_{0}^{\infty} d z \varphi(z, \xi)(1-\eta)^{*}(1-s)^{*}(z+\xi) n(\xi, \eta, t) n(z, s, t) \\
& \quad=\frac{1}{2} \iiint \int \cdots z \cdots+\frac{1}{2} \iiint \int \cdots \xi \cdots
\end{aligned}
$$

and each of the last two integrals coincides with the first integral on the righthand side of (1.8). Consequently

$$
\frac{\partial}{\partial t} \iint_{P} n=0
$$

and (1.6) follows. The proof of (1.7) is similar.

We next establish a stability result that will be needed later on.

Let $n_{\lambda}(x, y) \quad(0 \leq \lambda \leq 1)$ be a family of initial values satisfying:

$$
\begin{gathered}
n_{\lambda}(x, y) \text { is continuous and nonnegative for }(x, y) \in P, \\
\iint_{P}(1+x)\left|n_{\lambda}(x, y)-n_{0}(x, y)\right| \rightarrow 0 \quad \text { if } \lambda \rightarrow 0 .
\end{gathered}
$$

Denote by $n_{\lambda}(x, y, t)$ the solution of (1.1) with initial values $n_{\lambda}(x, y)$. 
Theorem 1.3. For any $T>0$,

$$
\iint_{P}(1+x)\left|n_{\lambda}(x, y, t)-n_{0}(x, y, t)\right| \rightarrow 0 \quad \text { as } \lambda \rightarrow 0
$$

uniformly in $t, 0 \leq t \leq T$.

Proof. Set

$$
\sigma_{\lambda}(t)=\iint_{P}(1+x)\left|n_{\lambda}(x, y, t)-n_{0}(x, y, t)\right| .
$$

Write (1.1), integrated in $t$, for $n_{\lambda}$ and $n_{0}$, and take the absolute value of the difference. Then, multiply by $(1+x)$ and integrate over $(x, y) \in P$. On the right-hand side we obtain integrals which can be estimated by terms of the form

$$
\int d t \iiint \int(1+x) \Phi_{i}\left|n_{\lambda}-n_{0}\right| n_{0}, \quad \int d t \iiint \int(1+x) \Phi_{i}\left|n_{\lambda}-n_{0}\right| n_{\lambda}
$$

where $\Phi_{1}\left(\Phi_{2}\right)$ corresponds to integrals obtained from $\int_{0}^{1} \int_{0}^{\infty}\left(\frac{1}{2} \int_{0}^{1} \int_{0}^{x}\right)$. Using Theorem 1.1 integrals with $i=1$ can be estimated directly, whereas for integrals with $i=2$ we first change the order of integration and change variables (cf. (1.9)). We then easily derive the inequality

$$
\sigma_{\lambda}(t) \leq C \int_{0}^{t} \sigma_{\lambda}(\tau) d \tau+\sigma_{\lambda}(0),
$$

from which we deduce that

$$
\sigma_{\lambda}(t) \leq C_{T} \sigma_{\lambda}(0) \text { if } 0 \leq t \leq T
$$

so that (1.11) follows.

\section{IMPENETRABLE SETS}

Defintion 2.1. A set $S$ in $P$ is called impenetrable if the impact of any two points not in $S$ is again not in $S$.

Example 1. Any set $\left\{y<y_{0}\right\}$ is impenetrable since if $(x, y) \oplus(\xi, \eta)=(z, s)$ then $s \geq \min \{y, \eta\}$. Analogously, $\left\{x<x_{0}\right\}$ is impenetrable.

Example 2. For any $C>0$, the set

$$
S=\left\{x \geq C y^{3}, 0<y<1\right\}
$$

is impenetrable. To prove this we have to show that if two points $(x, y),(\xi, \eta)$ satisfy $x<C y^{3}, \xi<C \eta^{3}$ then their impact $(z, s)$ satisfies

$$
s=\frac{y x^{2 / 3}+\eta \xi^{2 / 3}}{(x+\xi)^{2 / 3}}>\frac{(x+\xi)^{1 / 3}}{C^{1 / 3}}
$$

and this is obvious.

Example 3. The union of two impenetrable sets is impenetrable.

Theorem 2.1. If $S$ is impenetrable then the integral

$$
\iint_{S} n(x, y, t) d x d y
$$

is monotone decreasing in $t$. 
Proof. Integrating both sides of (1.1) over $S$ we get

$$
\begin{aligned}
& \frac{\partial}{\partial t} \iint_{S} n=-\int_{0}^{\infty} d x \int_{0}^{2^{1 / 3}} d y \int_{0}^{\infty} d \xi \int_{0}^{1} d \eta[\cdots] \chi_{S}(x, y) \\
& \quad+\frac{1}{2} \int_{0}^{\infty} d x \int_{0}^{2^{1 / 3}} d y \int_{0}^{\infty} d \xi \int_{0}^{1} d \eta[\cdots] \chi_{S}(x, y) \chi_{\{0 \leq \xi \leq x\}} .
\end{aligned}
$$

For any fixed $(\xi, \eta)$ define a transformation

$$
T_{\xi, \eta}:(x, y) \rightarrow(z, s)
$$

by

$$
z=x-\xi, \quad s=\frac{y x^{2 / 3}-\eta \xi^{2 / 3}}{z^{2 / 3}} .
$$

Then the second integral on the right-hand side of (2.2) can be written as

$$
\begin{aligned}
\int_{0}^{\infty} d \xi \int_{0}^{1} d \eta \int_{0}^{\infty} d z \int_{0}^{1} d s \varphi(\xi, z)(1-\eta)^{*}(1-s)^{*} \\
\times n(\xi, \eta, t) n(z, s, t) \chi_{\{z \geq 0\}} \chi_{S}(x, y)
\end{aligned}
$$

since

$$
\frac{\partial(x, y)}{\partial(z, s)}=\frac{1}{\partial s / \partial y}=\frac{z^{2 / 3}}{x^{2 / 3}}=\frac{(x-\xi)^{2 / 3}}{x^{2 / 3}} .
$$

The function $\chi_{S}(x, y)$ coincides with the function $\chi_{T_{\xi, \eta}(S)}(z, s)$, and $\chi_{\{z \geq 0\}} \equiv$ 1. Hence we get

$$
\iint_{P} d \xi d \eta \iint_{T_{\xi, \eta}(S)} \varphi(\xi, \eta)(1-\eta)^{*}(1-s)^{*} n(\xi, \eta, t) n(z, s, t) d z d s .
$$

Using this in (2.2) we find that

(2.3)

$$
\begin{aligned}
& \frac{\partial}{\partial t} \iint_{S} n(x, y, t) d x d y \\
& =-\iint_{P} d \xi d \eta \iint_{S}\left[\varphi(x, \xi)(1-y)^{*}(1-\eta)^{*} n(\xi, \eta, t) n(x, y, t)\right] d x d y \\
& \quad+\frac{1}{2} \iint_{P} d \xi d \eta \iint_{T_{\xi, \eta}(S)}\left[\varphi(\xi, z)(1-\eta)^{*}(1-s)^{*} n(\xi, \eta, t) n(z, s, t)\right] d z d s
\end{aligned}
$$

the integrands in the last two integrals are actually identical if we set $z=x$ and $s=y$ in the last integrand. Setting

(2.4) $H=\left\{(\xi, \eta, z, s) \in P \times P: T_{\xi, \eta}(x, y)=(z, s)\right.$ for some $\left.(x, y) \in S\right\}$, we shall prove that

$$
H \subset(P \times S) \cup(S \times P) ;
$$

it will then follow that the right-hand side in $(2.3)$ is $\leq 0$, and the theorem is proved.

To prove (2.5) let $(\xi, \eta, z, s) \in H$. Then by the definition of $T_{\xi, \eta}$,

$$
(\xi, \eta) \oplus(z, s)=(x, y) \text { for some }(x, y) \in S \text {. }
$$


Since $S$ is impenetrable, either $(\xi, \eta)$ or $(z, s)$ must be in $S$, and (2.5) follows.

Corollary 2.2. If $n_{0}(x, y)=0$ on an impenetrable set $S$, then $n(x, y, t)=0$ for $(x, y) \in S$ and all $t>0$.

By Example 2 above, for any $x_{0}>0, \gamma>0$, the set

$$
S=\left\{y<\frac{\gamma}{x_{0}^{1 / 3}} x^{1 / 3}\right\}
$$

is impenetrable. The set

$$
T \equiv\left\{0<x<x_{0}, \quad \gamma<y<1\right\}
$$

lies in the complement of $S$. Hence, by Corollary 2.2,

Corollary 2.3. If the support of $n_{0}(x, y)$ lies in a set as in (2.6) then, for any $t>0$, the support of $n(x, y, t)$ lies in

$$
\left\{x<\frac{x_{0}}{\gamma^{3}} y^{3}\right\} \text {. }
$$

In particular, $n(x, y, t) \equiv 0$ if $x \geq 2 x_{0} / \gamma^{3}$.

This means that if initially all the particles are partially painted at a uniformly positive fraction, then the size of the particles remains uniformly bounded for all times.

$$
\text { 3. } \int_{0}^{1} \int_{0}^{\infty} n d x d y \rightarrow 0 \text { IF } t \rightarrow \infty
$$

In this section we prove

Theorem 3.1. There holds

$$
\lim _{t \rightarrow \infty} \int_{0}^{1} \int_{0}^{\infty} n(x, y, t) d x d y=0 .
$$

This means that the number of particles which are not totally painted goes to zero as $t \rightarrow \infty$. (Compare with Theorem 4.1.)

We first establish

Lemma 3.2. For any $0<\theta<1$,

$$
\int_{0}^{\theta} \int_{0}^{\infty} n(x, y, t) d x d y \rightarrow 0 \quad \text { if } t \rightarrow \infty .
$$

Proof. Integrating $(1.1)$ over $(x, y) \in(0, \infty) \times(0,1)$ and proceeding similarly to the proof of (1.6) to change the order of integration and to change variables in the last integral on the right-hand side of (1.1), we obtain

$$
\begin{aligned}
& \frac{\partial}{\partial t} \int_{0}^{1} \int_{0}^{\infty} n(x, y, t) d x d y \\
& \leq-\frac{1}{2} \int_{0}^{1} \int_{0}^{\infty} \int_{0}^{1} \int_{0}^{\infty} \varphi(x, \xi)(1-\eta)^{*}(1-y)^{*} n(x, y, t) \\
& \times n(\xi, \eta, t) d x d y d \xi d \eta .
\end{aligned}
$$


By (1.3), we see that for any $\varepsilon>0$, there exists $c_{\varepsilon}>0$ such that $\varphi(x, \xi)>c_{\varepsilon}$ if $x, \xi \geq \varepsilon$. Using this in (3.3) we get

$$
\begin{aligned}
& \frac{\partial}{\partial t} \int_{0}^{1} \int_{0}^{\infty} n(x, y, t) d x d y \\
& \quad \leq-\frac{1}{2} c_{\varepsilon} \int_{0}^{\theta} \int_{\varepsilon}^{\infty} \int_{0}^{\theta} \int_{\varepsilon}^{\infty}(1-\eta)^{*}(1-y)^{*} n(\xi, \eta, t) n(x, y, t) \\
& \quad \leq-\frac{1}{2} c_{\varepsilon}(1-\theta)^{2}\left(\int_{0}^{\theta} \int_{\varepsilon}^{\infty} n(x, y, t) d x d y\right)^{2}
\end{aligned}
$$

Suppose (3.2) is not true; then, by Theorem 2.1 and Example 1,

$$
\int_{0}^{\theta} \int_{0}^{\infty} n(x, y, t) d x d y \downarrow \gamma>0 \text { as } t \uparrow \infty .
$$

Choose $\varepsilon$ small enough so that

$$
\int_{0}^{1} \int_{0}^{\varepsilon} n_{0}(x, y) d x d y<\frac{\gamma}{2} .
$$

Since the set $\{0<x<\varepsilon, 0<y<1\}$ is impenetrable (if $x>\varepsilon, \xi>\varepsilon$ then $x+\xi>\varepsilon)$,

$$
\int_{0}^{1} \int_{0}^{\varepsilon} n(x, y, t) d x d y<\frac{\gamma}{2} \text { for all } t>0
$$

(by Theorem 2.1) and therefore

$$
\int_{0}^{\theta} \int_{0}^{\varepsilon} n(x, y, t) d x d y<\frac{\gamma}{2} \quad \text { for all } t>0 .
$$

It follows that the integral on the right-hand side of $(3.4)$ is $\geq \frac{\gamma}{2}$ for all $t$ sufficiently large, so that

$$
\frac{\partial}{\partial t} \int_{0}^{1} \int_{0}^{\infty} n(x, y, t) d x d y \leq-\frac{1}{2} c_{\varepsilon}(1-\theta) \frac{\gamma^{2}}{4}
$$

This implies that $\int_{0}^{1} \int_{0}^{\infty} n$ becomes negative for $t$ large, a contradiction.

Proof of Theorem 3.1. By (1.6), for any $\varepsilon>0$ there exists an $x_{0}$ such that

$$
\int_{x_{0}}^{\infty} \int_{0}^{1} n(x, y, t) d x d y<\frac{C}{x_{0}}<\varepsilon .
$$

Choose $\xi_{0}$ positive and small enough such that

$$
\int_{0}^{\xi_{0}} \int_{0}^{1} n_{0}(x, y) d x d y<\frac{\varepsilon}{2}
$$

and choose $\delta$ positive and small such that

$$
\int_{\xi_{0}}^{2 \xi_{0}} \int_{1-\delta}^{1} n_{0}(x, y) d x d y<\frac{\varepsilon}{2^{2}} .
$$

Then, defining

$$
L=\left\{0<x<\xi_{0}, 0<y<1\right\} \cup\left\{\xi_{0}<x<2 \xi_{0}, 1-\delta<y<1\right\}
$$


we have

$$
\iint_{L} n_{0}(x, y) d x d y<\varepsilon\left(\frac{1}{2}+\frac{1}{2^{2}}\right) .
$$

Evidently $L$ is impenetrable, so that

$$
\iint_{L} n(x, y, t) d x d y<\varepsilon\left(\frac{1}{2}+\frac{1}{2^{2}}\right) \quad \forall t>0 .
$$

On the other hand, by Lemma 3.2,

$$
\int_{\xi_{0}}^{2 \xi_{0}} \int_{0}^{1-\delta} n(x, y, t) d x d y<\frac{\varepsilon}{2^{3}}
$$

if $t$ is sufficiently large, say, $t \geq t_{1}$. It follows that

$$
\int_{0}^{2 \xi_{0}} \int_{0}^{1} n(x, y, t) d x d y<\varepsilon\left(\frac{1}{2}+\frac{1}{2^{2}}+\frac{1}{2^{3}}\right) \quad \text { if } t \geq t_{1} .
$$

Next choose another small positive $\delta$ such that

$$
\int_{2 \xi_{0}}^{4 \xi_{0}} \int_{1-\delta}^{1} n\left(x, y, t_{1}\right) d x d y<\frac{\varepsilon}{2^{4}}
$$

and introduce the set

$$
L_{1}=\left\{0<x<2 \xi_{0}, 0<y<1\right\} \cup\left\{2 \xi_{0}<x<4 \xi_{0}, 1-\delta<y<1\right\} .
$$

This is again an impenetrable set. Since

$$
\iint_{L_{1}} n(x, y, t) d x d y<\varepsilon\left(\frac{1}{2}+\frac{1}{2^{2}}+\frac{1}{2^{3}}+\frac{1}{2^{4}}\right)
$$

for $t=t_{1}$, the inequality holds for all $t>t_{1}$. By Theorem 1.3,

$$
\int_{2 \xi_{0}}^{4 \xi_{0}} \int_{0}^{1-\delta} n(x, y, t) d x d y<\frac{\varepsilon}{2^{5}}
$$

if $t$ is sufficiently large, say $t \geq t_{2}$. Thus, together with (3.7),

$$
\int_{0}^{4 \xi_{0}} \int_{0}^{1} n(x, y, t) d x d y \leq \sum_{j=1}^{5} \frac{\varepsilon}{2^{j}} \quad \text { if } t \geq t_{2} .
$$

After a finite number of steps we conclude that

$$
\int_{0}^{x_{0}} \int_{0}^{1} n(x, y, t) d x d y<\varepsilon
$$

if $t$ is sufficiently large. Combining this with (3.5), the assertion (3.1) follows.

Set

From (1.1) we see that, if $y>1$, then $n(x, y, t)$ increases as $t$ increases.

$$
n_{\infty}(x, y) \equiv n(x, y, \infty)= \begin{cases}\lim _{t \rightarrow \infty} n(x, y, t), & y \geq 1, \\ 0, & 0 \leq y<1 .\end{cases}
$$

From Theorem 3.1 and the monotone convergence theorem we then have 
Theorem 3.3. As $t \rightarrow \infty, n(x, y, t) \rightarrow n_{\infty}(x, y)$ in $L^{1}(P)$.

$$
\text { 4. } \int_{0}^{1} \int_{0}^{\infty} x n(x, y, t) d x d y \rightarrow 0 \text { IF } t \rightarrow \infty
$$

In this section we consider the mass contained in particles which are not totally painted

$$
M(t)=\int_{0}^{1} \int_{0}^{\infty} x n(x, y, t) d x d y
$$

and prove

Theorem 4.1. $M(t) \rightarrow 0$ if $t \rightarrow \infty$.

Notice that (3.1) does not imply Theorem 4.1 since, in principle, a positive amount of mass could concentrate in a small number of very large and not completely painted particles. In order to prove the theorem we therefore resort to a more detailed description of the evolution of the system than the one given by (1.1).

Consider a particle $(x, y)$ at time $t$. Necessarily, there were, at $t=0$, particles $\left(\xi_{1}, \eta_{1}\right),\left(\xi_{2}, \eta_{2}\right),\left(\xi_{3}, \eta_{3}\right), \ldots$, that eventually combined to form $(x, y)$ at time $t$; that is to say,

$$
(x, y)=\left(\xi_{1}, \eta_{1}\right) \oplus\left(\xi_{2}, \eta_{2}\right) \oplus\left(\xi_{3}, \eta_{3}\right) \oplus \cdots .
$$

In order to track back in time the particles contributing to $(x, y)$, we define

$$
\begin{aligned}
& \left.\Lambda_{\xi, \eta}(x, y, f, t)=\text { number (density) of particles }(x, y) \text { (at time } t\right) \\
& \text { such that a fraction } f \text { of } x \text { consists of particles which were } \\
& \text { originally }(t=0) \text { of type }(\xi, \eta) .
\end{aligned}
$$

With this definition, the quantity

$$
\int_{1}^{2^{1 / 3}} \int_{0}^{\infty} \int_{0}^{1} f x \Lambda_{\xi, \eta}(x, y, f, t) d f d x d y
$$

is the total mass in $\{y>1\}$, at time $t$, originally contained in $(\xi, \eta)$-particles. Thus,

$$
\frac{1}{\xi} \int_{1}^{2^{1 / 3}} \int_{0}^{\infty} \int_{0}^{1} f x \Lambda_{\xi, \eta}(x, y, t) d f d x d y
$$

is the number of $(\xi, \eta)$-particles (at time $t=0$ ) whose mass is contained, at time $t$, in completely painted particles. Finally, if we set

$$
N(\xi, \eta, t)=n_{0}(\xi, \eta)-\frac{1}{\xi} \int_{1}^{2^{1 / 3}} \int_{0}^{\infty} \int_{0}^{1} f x \Lambda_{\xi, \eta}(x, y, f, t) d f d x d y
$$

then $N(\xi, \eta, t)$ represents the number of $(\xi, \eta)$-particles whose mass has remained in particles that are not totally painted, up to time $t$. We clearly have the "mass relation"

$$
\int_{0}^{1} \int_{0}^{\infty} x n(x, y, t) d x d y=\int_{0}^{1} \int_{0}^{\infty} \xi N(\xi, \eta, t) d \xi d \eta
$$


Also the total paint in particles with $y<1$ can be expressed either in terms of $n$ or in terms of $N$, so that

$$
\int_{0}^{1} \int_{0}^{\infty} x^{2 / 3} y n(x, y, t) d x d y=\int_{0}^{1} \int_{0}^{\infty} \xi^{2 / 3} \eta N(\xi, \eta, t) d \xi d \eta .
$$

From the considerations above we should also have that

$$
N \geq 0
$$

and that $\Lambda_{\xi, \eta}$ increases with time for $y>1$, so

$$
N(\xi, \eta, t) \downarrow \quad \text { if } t \uparrow
$$

We now have

Lemma 4.2. If there exists a measurable function $N(\xi, \eta, t)$ defined for $0 \leq$ $\xi<\infty, 0 \leq \eta \leq 1, t \geq 0$ and satisfying (4.3)-(4.6) then

$$
\int_{0}^{1} \int_{0}^{\infty} x n(x, y, t) d x d y \rightarrow 0 \quad \text { if } t \rightarrow \infty .
$$

Proof. Set $N_{0}(\xi, \eta)=\lim _{t \rightarrow \infty} N(\xi, \eta, t)$. By (1.6) and Theorem 3.1

$$
\int_{0}^{1} \int_{0}^{\infty} x^{2 / 3} n(x, y, t) d x d y \leq\left(\int_{0}^{1} \int_{0}^{\infty} x n\right)^{2 / 3}\left(\int_{0}^{1} \int_{0}^{\infty} n\right)^{1 / 3} \rightarrow 0
$$

as $t \rightarrow \infty$. Hence also

$$
\lim _{t \rightarrow \infty} \int_{0}^{1} \int_{0}^{\infty} x^{2 / 3} y n(x, y, t) d x d y=0 .
$$

From (4.4) and the monotone convergence theorem we then deduce that

$$
\int_{0}^{1} \int_{0}^{\infty} \xi^{2 / 3} \eta N_{0}(\xi, \eta) d \xi d \eta=0
$$

hence $N_{0}=0$. By the monotone convergence we then also have

$$
\lim _{t \rightarrow \infty} \int_{0}^{1} \int_{0}^{\infty} \xi N(\xi, \eta, t) d \xi d \eta=0
$$

and, upon recalling (4.3), the conclusion (4.7) follows.

To prove rigorously that a function $N$ as above in fact does exist, we shall first write down the evolution equation for $\Lambda_{\xi, \eta}$. The number $\Lambda_{\xi, \eta}(x, y, f, t)$ decreases by collision of particles $(x, y)$ with any particle in the system; on the other hand, it increases as a result of collisions of particles $\left(x^{\prime}, y^{\prime}\right)$ and $\left(x-x^{\prime}, s\right)$ containing $(\xi, \eta)$-particles in fractions $f^{\prime}$ and $\frac{f x-f^{\prime} x^{\prime}}{\left(x-x^{\prime}\right)}$, respectively. 
Thus, $\Lambda_{\xi, \eta}$ must verify the following equation

$$
\begin{aligned}
& \frac{\partial}{\partial t} \Lambda_{\xi, \eta}(x, y, f, t)=-\Lambda_{\xi, \eta}(x, y, f, t) \int_{0}^{\infty} d x^{\prime} \\
& \quad \times \int_{0}^{1} d y^{\prime}\left(1-y^{\prime}\right)(1-y) \varphi\left(x^{\prime}, x\right) n\left(x^{\prime}, y^{\prime}, t\right) \\
& \quad+\frac{1}{2} \int_{0}^{x} d x^{\prime} \int_{0}^{1} d y^{\prime} \int_{0}^{1} d f^{\prime} \Lambda_{\xi, \eta}\left(x^{\prime}, y^{\prime}, f^{\prime}, t\right) \\
& \quad \times \Lambda_{\xi, \eta}\left(x-x^{\prime}, s, \frac{f x-f^{\prime} x^{\prime}}{x-x^{\prime}}, t\right)\left(1-y^{\prime}\right)^{*}(1-s)^{*} \\
& \quad \times \varphi\left(x-x^{\prime}, x^{\prime}\right) \frac{x^{2 / 3}}{\left(x-x^{\prime}\right)^{2 / 3}} \frac{x}{x-x^{\prime}} .
\end{aligned}
$$

We would also like to determine initial conditions for $\Lambda$, to prove that there exists a solution to (4.8) with these initial conditions, and to verify the relations (4.3)-(4.6) for $N$ defined by (4.2). However $\Lambda$ is a complicated mathematical object, which is "singular" at $f=0$ (for example if $t=0$ ).

To circumvent this difficulty we can work instead with a more regular "function:"

$$
K_{\xi, \eta}(x, y, t)=\int_{0}^{1} f \Lambda_{\xi, \eta}(x, y, f, t) d f .
$$

Indeed, from (4.2),

$$
N(\xi, \eta, t)=n_{0}(\xi, \eta)-\frac{1}{\xi} \int_{1}^{2^{1 / 3}} \int_{0}^{\infty} x K_{\xi, \eta}(x, y, t) d x d y .
$$

Of course, so far everything is formal; rigorous proofs will be given below.

If we integrate both sides of (4.8) with respect to $f d f$, we get

$$
\begin{aligned}
& \frac{\partial K_{\xi, \eta}(x, y, t)}{\partial t}=-K_{\xi, \eta}(x, y, t) \\
& \quad \times \int_{0}^{\infty} d x^{\prime} \int_{0}^{1} d y^{\prime}\left(1-y^{\prime}\right)(1-y) \varphi\left(x^{\prime}, x\right) n\left(x^{\prime}, y^{\prime}, t\right) \\
& \quad+\frac{1}{2} \int_{0}^{x} d x^{\prime} \int_{0}^{1} d y^{\prime} \int_{0}^{1} d f^{\prime} \int_{0}^{1} f d f \frac{x}{x-x^{\prime}} \Lambda_{\xi, \eta}\left(x^{\prime}, y^{\prime}, f^{\prime}, t\right) \\
& \quad \times \Lambda_{\xi, \eta}\left(x-x^{\prime}, s, \frac{f x-f^{\prime} x^{\prime}}{x-x^{\prime}}, t\right) \\
& \quad \times\left(1-y^{\prime}\right)^{*}(1-s)^{*} \varphi\left(x-x^{\prime}, x^{\prime}\right) \frac{x^{2 / 3}}{\left(x-x^{\prime}\right)^{2 / 3}} .
\end{aligned}
$$

To integrate on $f$ in the last integral we change variables

$$
f=\frac{q\left(x-x^{\prime}\right)+f^{\prime} x^{\prime}}{x}, \quad d f \frac{x}{x-x^{\prime}}=d q ;
$$


the last integral becomes

$$
\begin{aligned}
& \int_{0}^{x} d x^{\prime} \int_{0}^{1} d y^{\prime} \frac{x-x^{\prime}}{x} \int_{0}^{1} d f^{\prime} \Lambda_{\xi, \eta}\left(x^{\prime}, y^{\prime}, f^{\prime}, t\right) K_{\xi, \eta}\left(x-x^{\prime}, s, t\right) \\
& \quad \times\left(1-y^{\prime}\right)^{*}(1-s)^{*} \varphi\left(x-x^{\prime}, x^{\prime}\right) \frac{x^{2 / 3}}{\left(x-x^{\prime}\right)^{2 / 3}} \\
& \quad+\int_{0}^{x} d x^{\prime} \int_{0}^{1} d y^{\prime} \frac{x^{\prime}}{x} \int_{0}^{1} d q \Lambda_{\xi, \eta}\left(x-x^{\prime}, s, q, t\right) K_{\xi, \eta}\left(x^{\prime}, y^{\prime}, t\right) \\
& \quad \times\left(1-y^{\prime}\right)^{*}(1-s)^{*} \varphi\left(x-x^{\prime}, x^{\prime}\right) \frac{x^{2 / 3}}{\left(x-x^{\prime}\right)^{2 / 3}} .
\end{aligned}
$$

Formally

$$
n\left(x^{\prime}, y^{\prime}, t\right)=\int_{0}^{1} d f^{\prime} \Lambda_{\xi, \eta}\left(x^{\prime}, y^{\prime}, f^{\prime}, t\right),
$$

since $(x, y)$ always contains some fraction of $(\xi, \eta)$ (possibly zero fraction). Using this remark to simplify the preceding two integrals, we then obtain from (4.11),

$$
\begin{aligned}
& \frac{\partial K_{\xi, \eta}(x, y, t)}{\partial t}=-K_{\xi, \eta}(x, y, t) \int_{0}^{\infty} d x^{\prime} \int_{0}^{1} d y^{\prime} \\
& \quad \times\left(1-y^{\prime}\right)^{*}(1-y)^{*} \varphi\left(x^{\prime}, x\right) n\left(x^{\prime}, y^{\prime}, t\right) \\
& +\frac{1}{2} \int_{0}^{x} d x^{\prime} \int_{0}^{1} d y^{\prime}\left[\frac{x-x^{\prime}}{x} n\left(x^{\prime}, y^{\prime}, t\right) K_{\xi, \eta}\left(x-x^{\prime}, s, t\right)\right. \\
& \left.\quad+\frac{x^{\prime}}{x} n\left(x-x^{\prime}, s, t\right) K_{\xi, \eta}\left(x^{\prime}, y^{\prime}, t\right)\right] \\
& \times\left(1-y^{\prime}\right)^{*}(1-s)^{*} \varphi\left(x-x^{\prime}, x^{\prime}\right) \frac{x^{2 / 3}}{\left(x-x^{\prime}\right)^{2 / 3}},
\end{aligned}
$$

where

$$
y^{\prime} x^{\prime 2 / 3}+s\left(x-x^{\prime}\right)^{2 / 3}=y x^{2 / 3}
$$

Notice that, upon a change of variables, (4.13a) can be written as

$$
\begin{aligned}
& \frac{\partial K_{\xi, \eta}(x, y, t)}{\partial t}=-K_{\xi, \eta}(x, y, t) \int_{0}^{\infty} d x^{\prime} \int_{0}^{1} d y^{\prime} \\
& \quad \times\left(1-y^{\prime}\right)^{*}(1-y)^{*} \varphi\left(x^{\prime}, x\right) n\left(x^{\prime}, y^{\prime}, t\right) \\
& \quad+\int_{0}^{x} d x^{\prime} \int_{0}^{1} d y^{\prime} \frac{x^{\prime}}{x} n\left(x-x^{\prime}, s, t\right) K_{\xi, \eta}\left(x^{\prime}, y^{\prime}, t\right) \\
& \quad \times\left(1-y^{\prime}\right)^{*}(1-s)^{*} \varphi\left(x-x^{\prime}, x^{\prime}\right) \frac{x^{2 / 3}}{\left(x-x^{\prime}\right)^{2 / 3}}
\end{aligned}
$$

Further, since

$$
\begin{aligned}
\int_{W} d \xi & d \eta \int_{V} d x d y x K_{\xi, \eta}(x, y, 0) \\
& =\{\text { mass contained in } V \text { at } t=0 \text { which is in } W \text { at } t=0\} \\
& =\int_{V \cap W} x n_{0}(x, y) d x d y,
\end{aligned}
$$


we see that

$$
K_{\xi, \eta}(x, y, 0)=\delta(x-\xi) \delta(y-\eta) n_{0}(x, y) .
$$

One can show that the system (4.13), (4.15) has a measure-valued solution. However, in order to prove (4.3)-(4.6) it is much easier to work with smooth functions $K_{\xi, \eta}^{m}$ which are approximations to $K_{\xi, \eta}$. The functions $K_{\xi, \eta}^{m}$ will solve (4.13) with initial conditions

$$
K_{\xi, \eta}^{m}(x, y, 0)=\delta_{m}(x-\xi) \delta_{m}(y-\eta) n_{0}(x, y) \chi_{y>\frac{1}{m}} \chi_{m>\xi>\frac{1}{m}}
$$

where $\delta_{m}(t)$ is a smooth approximation to the Dirac measure $\delta(t)$, i.e. "an approximation to the identity:"

$$
\delta_{m}(t)=\theta(m t) m,
$$

where

$$
\theta \in C_{0}^{\infty}(-1,1), \quad 0 \leq \theta \leq 1, \quad \int_{\mathbb{R}} \theta=1,
$$

and $\chi_{A}$ is the characteristic function of the set $A$.

Lemma 4.3. There exists a unique solution $K_{\xi, \eta}^{m}(x, y, t)$ of (4.13), (4.16) which is continuous in $(\xi, \eta, x, y, t)$. Furthermore,

$$
K_{\xi, \eta}^{m} \equiv 0 \quad \text { if } \xi \leq \frac{1}{m} \quad \text { or } \quad \xi \geq m
$$

and

$$
\sup _{t \leq T} \int_{0}^{2^{1 / 3}} \int_{0}^{\infty}(1+x) K_{\xi, \eta}^{m}(x, y, t) d x d y \leq C_{T} .
$$

Proof. The proof of existence and uniqueness of a solution satisfying (4.18) can be established by the method of successive approximations. Uniqueness (for each $(\xi, \eta)$ ) and (4.16) clearly implies (4.17). To prove that $K_{\xi, \eta}^{m}$ is nonnegative, write the second term in the right-hand side of $(4.13 \mathrm{~b})$ as

$$
\iint_{\Delta(x)} K_{\xi, \eta}\left(x^{\prime}, y^{\prime}, t\right) B\left(x, x^{\prime}, y, y^{\prime}, t\right) d x^{\prime} d y^{\prime}
$$

where $\Delta(x)=\left\{\left(x^{\prime}, y^{\prime}\right): 0 \leq x^{\prime} \leq x, 0 \leq y^{\prime} \leq 1\right\}$.

We now replace $\Delta(x)$ by

$$
\Delta_{M}(x)= \begin{cases}\Delta(x), & x \leq M, \\ \Delta(M), & x>M,\end{cases}
$$

and add the number $\frac{1}{M}$ to the right-hand side of (4.16).

Denote the solution corresponding to this problem (with $0 \leq x \leq M$ ) by $K_{\xi, \eta}^{m, M}(x, y, t)$. One can easily show that, for any fixed $(x, y, t)$,

$$
K_{\xi, \eta}^{m, M}(x, y, t) \rightarrow K_{\xi, \eta}^{m}(x, y, t) \quad \text { as } M \rightarrow \infty .
$$

Therefore it is sufficient to prove that $K_{\xi, \eta}^{m, M} \geq 0$. The function $\psi(x, y, t) \equiv$ $K_{\xi, \eta}^{m, M}(x, y, t)$ satisfies (for $\left.0 \leq x \leq M, 0 \leq y \leq 1\right)$ an equation of the form

$$
\frac{\partial \psi}{\partial t}+a \psi=f, \quad \psi(x, y, 0)>0
$$


where $f(x, y, 0)>0$ and $f(x, y, s) \geq 0$ for all $0 \leq s \leq t$ if $\psi(x, y, s) \geq 0$ for all $0 \leq s \leq t$. A simple argument shows that $\psi(x, y, t)$ must then remain strictly positive for all $t>0$. 4.4).

The following two lemmas will be used later on for establishing (4.3) and

Lemma 4.4. Let $n^{m}$ denote the solution of (1.1) with initial values

$$
n^{m}(x, y, 0)=n_{0}^{m}(x, y) \equiv \int_{0}^{1} d \eta \int_{0}^{\infty} d \xi K_{\xi, \eta}^{m}(x, y, 0) .
$$

Then

$$
n^{m}(x, y, t) \equiv \int_{0}^{1} d \eta \int_{0}^{\infty} d \xi K_{\xi, \eta}^{m}(x, y, t)
$$

\section{furthermore}

(4.20) $\quad n^{m} \rightarrow n$ in $L^{1}((1+x) d x d y)$ as $m \rightarrow \infty$, uniformly for $0 \leq t \leq T$.

Proof. Denote the right-hand side of $(4.19)$ by $\Gamma(x, y, t)$. Integrating both sides of (4.13a) (for $K_{\xi, \eta}^{m}$ ) with respect to $(\xi, \eta)$ we see that $\Gamma$ satisfies the same evolution equation as $K_{\xi, \eta}^{m}$. Since $n^{m}$ also satisfies (4.13a) and $n^{m}(x, y, 0) \equiv \Gamma(x, y, 0),(4.19)$ follows by the uniqueness of solutions to (4.13).

Using (4.16), we can write

$$
\begin{aligned}
n_{0}^{m}(x, y) & =\left(\int_{0}^{1} d \eta \int_{0}^{\infty} d \xi \delta_{m}(x-\xi) \delta_{m}(y-\eta) \chi_{y>\frac{1}{m}} \chi_{m>\xi>\frac{1}{m}}\right) n_{0}(x, y) \\
& \equiv \psi_{m}(x, y) n_{0}(x, y) .
\end{aligned}
$$

Then

$$
\begin{aligned}
\psi_{m}(x, y) & =\int_{0}^{1} d \eta \int_{0}^{\infty} d \xi m \theta(m(x-\xi)) m \theta(m(y-\eta)) \chi_{y>\frac{1}{m}} \chi_{m>\xi>\frac{1}{m}} \\
& =\left(\int_{-(1-y) m}^{m y} \theta(s) \chi_{y>\frac{1}{m}} d s\right)\left(\int_{-\infty}^{m x} \theta(z) \chi_{m>x-z / m>\frac{1}{m}} d z\right) \\
& =\left(\chi_{y>\frac{1}{m}} \int_{-(1-y) m}^{m y} \theta(s) d s\right)\left(\int_{-m^{2}+m x}^{m x-1} \theta(z) d z\right)
\end{aligned}
$$

so that, for $0<y<1, x>0$,

$$
0 \leq \psi_{m} \leq 1 \quad \text { and } \quad \psi_{m} \rightarrow 1 \quad \text { as } m \rightarrow \infty .
$$

Therefore, the dominated convergence theorem implies

$$
n_{0}^{m} \rightarrow n_{0} \quad \text { in } L^{1}((1+x) d x d y) \quad \text { as } m \rightarrow \infty
$$

and this in turns implies (4.20), upon using Theorem 1.3.

A similar argument can be used to establish 
Lemma 4.5. Let $\widetilde{n^{m}}$ denote the solution of (1.1) with initial values

$$
\widetilde{n^{m}}(x, y, 0)=\widetilde{n_{0}^{m}}(x, y) \equiv \int_{0}^{1} d \eta \int_{0}^{\infty} d \xi^{x^{1 / 3}} \frac{\eta}{\xi^{1 / 3}} \frac{{ }^{m}}{y} K_{\xi, \eta}^{m}(x, y, 0) \text {. }
$$

Then

$$
\widetilde{n^{m}}(x, y, t) \equiv \int_{0}^{1} d \eta \int_{0}^{\infty} d \xi \frac{x^{1 / 3}}{\xi^{1 / 3}} \frac{\eta}{y} K_{\xi, \eta}^{m}(x, y, t)
$$

furthermore

$$
\begin{array}{r}
\widetilde{n^{m}}(x, y, t) \rightarrow n(x, y, t) \text { in } L^{1}((1+x) d x d y) \text { as } m \rightarrow \infty, \\
\text { uniformly for } 0 \leq t \leq T .
\end{array}
$$

Next, define (cf. (4.10))

$$
N^{m}(\xi, \eta, t) \equiv \overline{n_{0}^{m}}(\xi, \eta)-\frac{1}{\xi} \int_{0}^{\infty} d x \int_{1}^{2^{1 / 3}} d y x K_{\xi, \eta}^{m}(x, y, t)
$$

where

$$
\overline{n_{0}^{m}}(\xi, \eta) \equiv \frac{1}{\xi} \int_{0}^{\infty} d x \int_{0}^{2^{1 / 3}} d y x K_{\xi, \eta}^{m}(x, y, 0)
$$

Lemma 4.6. The functions $N^{m}, \overline{n_{0}^{m}}$ satisfy

(i) $N^{m} \geq 0$,

(ii) $N^{m}(x, y, t)$ is nonincreasing in $t$ for $(x, y)$ fixed,

(iii) $\overline{n_{0}^{m}} \rightarrow n_{0}$ in $L^{1}((1+x) d x d y)$ as $m \rightarrow \infty$.

Proof. To prove (i), it suffices to show that

$$
\int_{0}^{\infty} d x \int_{0}^{2^{1 / 3}} d y x K_{\xi, \eta}^{m}(x, y, t) \equiv \xi \overline{n_{0}^{m}}(\xi, \eta) .
$$

Denoting the left-hand side of $(4.25)$ by $\mu(t),(4.25)$ will follow from (4.24) once we prove that

$$
\mu^{\prime}(t) \equiv 0
$$

Multiplying (4.13b) by $x$ and integrating in $(x, y)$ we obtain

$$
\begin{aligned}
\mu^{\prime}(t)= & -\int_{0}^{\infty} d x \int_{0}^{2^{1 / 3}} d y x K_{\xi, \eta}^{m}(x, y, t) \int_{0}^{\infty} d x^{\prime} \int_{0}^{1} d y^{\prime} \\
& \times\left(1-y^{\prime}\right)^{*}(1-y)^{*} \varphi\left(x, x^{\prime}\right) n\left(x^{\prime}, y^{\prime}, t\right) \\
& +\int_{0}^{\infty} d x \int_{0}^{2^{1 / 3}} d y \int_{0}^{x} d x^{\prime} \int_{0}^{1} d y^{\prime} x^{\prime} n\left(x-x^{\prime}, s, t\right) \\
& \times K_{\xi, \eta}^{m}\left(x^{\prime}, y^{\prime}, t\right)\left(1-y^{\prime}\right)(1-s)^{*} \varphi\left(x-x^{\prime}, x^{\prime}\right) \frac{x^{2 / 3}}{\left(x-x^{\prime}\right)^{2 / 3}} \\
\equiv & -I_{1}+I_{2} .
\end{aligned}
$$

Now, once again we use Fubini's theorem and a change of variables to show that $I_{1}=I_{2}$, thereby establishing (4.26).

From (4.13) it is clear that

$$
\frac{\partial K_{\xi, \eta}^{m}}{\partial t}(x, y, t) \geq 0 \text { if } y>1
$$

and (ii) follows. 
Finally, we want to prove (iii). Making a change of variables, we get

$$
\begin{aligned}
\overline{n_{0}^{m}}(\xi, \eta)= & \frac{1}{\xi} \int_{-\xi m}^{\infty} d z \int_{-\eta m}^{\left(2^{1 / 3}-\eta\right) m} d s\left(\xi+\frac{z}{m}\right) \chi_{\eta+s / m>\frac{1}{m}} \chi_{m>\xi>\frac{1}{m}} \\
& \times \theta(z) \theta(s) n_{0}\left(\xi+\frac{z}{m}, \eta+\frac{s}{m}\right)
\end{aligned}
$$

Hence,

$$
\begin{aligned}
& \int_{0}^{\infty} d \xi \int_{0}^{2^{1 / 3}} d \eta(1+\xi)\left|\overline{n_{0}^{m}}(\xi, \eta)-n_{0}(\xi, \eta)\right| \\
& \leq \int_{0}^{\infty} \int_{0}^{2^{1 / 3}} d \xi d \eta(1+\xi) \int_{-\xi m}^{\infty} d z \int_{-\eta m}^{\left(2^{1 / 3}-\eta\right) m} d s(1+z / m \xi) \\
& \quad \times \chi_{\eta+s / m>\frac{1}{m}} \chi_{m>\xi>\frac{1}{m}} \theta(z) \theta(s)\left|n_{0}(\xi+z / m, \eta+s / m)-n_{0}(\xi, \eta)\right| \\
& \quad+\int_{0}^{\infty} \int_{0}^{2^{1 / 3}} d \xi d \eta(1+\xi) n_{0}(\xi, \eta) \mid \int_{-\xi m}^{\infty} d z \int_{-\eta m}^{\left(2^{1 / 3}-\eta\right) m} d s(1+z / m \xi) \\
& \equiv J_{1}^{m}+J_{2}^{m} .
\end{aligned}
$$

But, clearly

$$
\begin{aligned}
J_{1}^{m} \leq & 2 \int_{-\infty}^{\infty} d z \int_{-\infty}^{\infty} d s \theta(z) \theta(s) \int_{0}^{\infty} d \xi \int_{0}^{2^{1 / 3}} d \eta(1+\xi) \\
& \times\left|n_{0}(\xi+z / m, \eta+s / m)-n_{0}(\xi, \eta)\right| \rightarrow 0
\end{aligned}
$$

as $m \rightarrow \infty$ and

$$
J_{2}^{m} \rightarrow 0 \quad \text { as } m \rightarrow \infty \text {. }
$$

Next, we estimate the "mass" and "paint" for the functions $N^{m}$.

Lemma 4.7. For any $T>0$,

$$
\int_{0}^{\infty} d \xi \int_{0}^{1} d \eta \xi N^{m}(\xi, \eta, t)=\int_{0}^{\infty} d x \int_{0}^{1} d y x n(x, y, t)+\sigma_{m}(t)
$$

where $\sigma_{m}(t) \rightarrow 0$ as $m \rightarrow \infty$, uniformly for $0 \leq t \leq T$.

Proof. Integrating (4.23) and using (4.19), we get

$$
\begin{aligned}
\int_{0}^{\infty} & d \xi \int_{0}^{1} d \eta \xi N^{m}(\xi, \eta, t)=\int_{0}^{\infty} d \xi \int_{0}^{1} d \eta \xi \overline{n_{0}^{m}}(\xi, \eta) \\
& -\int_{0}^{\infty} d \xi \int_{0}^{1} d \eta \int_{0}^{\infty} d x \int_{1}^{2^{1 / 3}} d y x K_{\xi, \eta}^{m} \\
= & \int_{0}^{\infty} d \xi \int_{0}^{1} d \eta \xi \overline{n_{0}^{m}}-\int_{0}^{\infty} d x \int_{1}^{2^{1 / 3}} d y x n^{m}(x, y, t)
\end{aligned}
$$


Now, from (1.6), we know that

$$
\begin{aligned}
\int_{0}^{\infty} & d x \int_{1}^{2^{1 / 3}} d y x n^{m}(x, y, t) \\
& =\int_{0}^{\infty} d x \int_{0}^{1} d y x n_{0}^{m}(x, y)-\int_{0}^{\infty} d x \int_{0}^{1} d y x n^{m}(x, y, t) .
\end{aligned}
$$

Hence,

$$
\int_{0}^{\infty} d \xi \int_{0}^{1} d \eta \xi N^{m}(\xi, \eta, t)=\int_{0}^{\infty} d x \int_{0}^{1} d y x n(x, y, t)+\sigma_{m}(t)
$$

where

$$
\begin{aligned}
\sigma_{m}(t)= & \left.\int_{0}^{\infty} d \xi \int_{0}^{1} d \eta \xi \overline{n_{0}^{m}}(\xi, \eta)-n_{0}(\xi, \eta)\right) \\
& +\int_{0}^{\infty} d \xi \int_{0}^{1} d \eta \xi\left(n_{0}(\xi, \eta)-n_{0}^{m}(\xi, \eta)\right) \\
& +\int_{0}^{\infty} d x \int_{0}^{1} d y x\left(n^{m}(x, y, t)-n(x, y, t)\right) .
\end{aligned}
$$

Finally, (4.20) and Lemma 4.6(iii) imply

$$
\sigma_{m}(t) \rightarrow 0 \text { as } m \rightarrow \infty, \text { uniformly for } t \leq T
$$

as desired.

In a similar way, but using Lemma 4.5 instead of Lemma 4.4 , one can prove

\section{Lemma 4.8.}

$$
\int_{0}^{\infty} d \xi \int_{0}^{1} d \eta \xi^{2 / 3} \eta N^{m}(\xi, \eta, t)=\int_{0}^{\infty} d x \int_{0}^{1} d y x^{2 / 3} y n(x, y, t)+\gamma_{m}(t)
$$

where $\gamma_{m}(t) \rightarrow 0$ as $m \rightarrow \infty$, uniformly for $0 \leq t \leq T$.

The existence of a function $N$ is established in the following

Theorem 4.9. There exists a function $N(x, y, t)$ such that, for any $T>0$,

$$
\begin{array}{r}
N^{m}(\cdot, t) \rightarrow N(\cdot, t) \text { in } L^{1}((1+x) d x d y) \\
\text { as } m \rightarrow \infty, \text { uniformly for } 0 \leq t \leq T .
\end{array}
$$

Before giving the proof of the theorem, we notice that $N$ is the desired function, that is

Corollary 4.10. The function $N$ in Theorem 4.9 satisfies (4.3)-(4.6). 4.9.

The corollary is an immediate consequence of Lemmas 4.6-4.8 and Theorem

Proof of Theorem 4.9. Set

$$
\Gamma^{m}(\xi, \eta, t) \equiv \frac{1}{\xi} \int_{0}^{\infty} d x \int_{1}^{2^{1 / 3}} d y x K_{\xi, \eta}^{m}(x, y, t)
$$

By (4.23) and Lemma 4.6(iii), (4.29) will follow if we show that

$$
\left\{\Gamma^{m}(\cdot, t)\right\} \text { converges in } L^{1}((1+\xi) d \xi d \eta)
$$


Clearly, (4.13) and Gronwall's inequality imply, for $t \leq T$,

$$
\begin{aligned}
\int_{0}^{\infty} & d \xi \int_{0}^{2^{1 / 3}} d \eta(1+\xi)\left|\Gamma^{j}(\xi, \eta, t)-\Gamma^{k}(\xi, \eta, t)\right| \\
\leq & C_{T} \int_{0}^{\infty} d \xi \int_{0}^{2^{1 / 3}} d \eta \frac{(1+\xi)}{\xi} \\
& \times \int_{0}^{\infty} d x \int_{0}^{2^{1 / 3}} d y x\left|K_{\xi, \eta}^{j}(x, y, 0)-K_{\xi, \eta}^{k}(x, y, 0)\right|
\end{aligned}
$$

so that it suffices to show that the right-hand side of (4.31) converges to zero as $j, k \rightarrow \infty$. Since

$$
\begin{aligned}
\int_{0}^{\infty} & d \xi \int_{0}^{2^{1 / 3}} d \eta \frac{(1+\xi)}{\xi} \int_{0}^{\infty} d x \int_{0}^{2^{1 / 3}} d y x\left|K_{\xi, \eta}^{j}(x, y, 0)-K_{\xi, \eta}^{k}(x, y, 0)\right| \\
= & \int_{0}^{\infty} d x \int_{0}^{2^{1 / 3}} d y x n_{0}(x, y) \\
& \times\left\{\int_{0}^{\infty} d \xi \int_{0}^{2^{1 / 3}} d \eta \frac{(1+\xi)}{\xi} \mid \delta_{j}(x-\xi) \delta_{j}(y-\eta) \chi_{y>\frac{1}{j}} \chi_{j>\xi>\frac{1}{j}}\right. \\
& \left.-\delta_{k}(x-\xi) \delta_{k}(y-\eta) \chi_{y>\frac{1}{k}} \chi_{k>\xi>\frac{1}{k}} \mid\right\} \\
\equiv & \int_{0}^{\infty} d x \int_{0}^{2^{1 / 3}} d y x n_{0}(x, y) \int_{0}^{\infty} d \xi \int_{0}^{2^{1 / 3}} d \eta \frac{(1+\xi)}{\xi}\left|\Delta_{j}-\Delta_{k}\right|
\end{aligned}
$$

it is enough to prove that

$$
x \int_{0}^{\infty} d \xi \int_{0}^{2^{1 / 3}} d \eta \frac{(1+\xi)}{\xi}\left|\Delta_{j}-\Delta_{k}\right| \equiv I_{j k} \leq C(1+x)
$$

for some constant $C>0$, and that

$$
I_{j k} \rightarrow 0 \text { as } j, k \rightarrow \infty .
$$

The inequality (4.32) is an immediate consequence of

$$
\begin{aligned}
& x \int_{0}^{\infty} d \xi \int_{0}^{2^{1 / 3}} d \eta \frac{(1+\xi)}{\xi} \Delta_{j} \\
& \quad=\int_{-(1-y) j}^{y j} d s \int_{-\left(j^{2}-j x\right)}^{x j-1} d z \theta(z) \theta(s) \chi_{j>x-z / j>\frac{1}{j}}\left(\frac{1}{(1-z / j x)}+x\right)
\end{aligned}
$$

while (4.33) can be easily proved using (4.34).

\section{ASYMPTOTIC STABILITY}

Using Theorem 4.1 we can extend the stability result in Theorem 1.3 to the case $t=\infty$ :

Theorem 5.1. Under the assumption (1.10),

$$
\int_{1}^{2^{1 / 3}} d y \int_{0}^{\infty} x\left|n_{\lambda}^{\infty}(x, y)-n_{0}^{\infty}(x, y)\right| d x \rightarrow 0 \quad \text { as } \lambda \rightarrow 0 .
$$


Here $n_{\lambda}^{\infty}(x, y)$ is the $L^{1}(P)$-limit of $n_{\lambda}(x, y, t)$ as $t \rightarrow \infty$.

Proof. Consider the family of functions, parameterized by $t$ :

$$
f_{t}(\lambda)=\int_{0}^{1} d y \int_{0}^{\infty} x n_{\lambda}(x, y, t) d x, \quad 0 \leq \lambda \leq 1 .
$$

By Theorem 1.3, $f_{t}(\lambda) \rightarrow f_{t}(0)$ as $\lambda \rightarrow 0$, for any $t>0$. Since $\partial n_{\lambda}(x, y, t) / \partial t$ $\geq 0$ if $y>1$, it follows from (1.6) that

$$
f_{t}(\lambda) \downarrow \quad \text { if } t \uparrow .
$$

By Theorem 4.1

$$
\lim _{t \rightarrow \infty} f_{t}(\lambda)=0 \text { for any } \lambda .
$$

We can therefore apply Dini's lemma to conclude that given $\varepsilon>0$ there exist $\lambda_{0}>0$ and $T>0$ such that, for all $0 \leq \lambda \leq \lambda_{0}$, we have $f_{t}(\lambda)<\varepsilon$ if $t \geq T$. By the conservation of mass and since $n_{\lambda}^{\infty}(x, y) \geq n_{\lambda}(x, y, t)$ if $y>1$, it follows that

$$
\begin{aligned}
\int_{1}^{2^{1 / 3}} \int_{0}^{\infty} x\left|n_{\lambda}^{\infty}(x, y)-n_{\lambda}(x, y, t)\right| d x d y<\varepsilon \\
\text { if } t \geq T \text { and } 0 \leq \lambda \leq \lambda_{0} .
\end{aligned}
$$

We can now estimate the left-hand side of $(5.1)$ by

$$
\begin{aligned}
\int_{1}^{2^{1 / 3}} & \int_{0}^{\infty} x\left|n_{\lambda}^{\infty}(x, y)-n_{\lambda}(x, y, T)\right| \\
& +\int_{1}^{2^{1 / 3}} \int_{0}^{\infty} x\left|n_{\lambda}(x, y, T)-n_{0}(x, y, T)\right| \\
& +\int_{1}^{2^{1 / 3}} \int_{0}^{\infty} x\left|n_{0}(x, y, T)-n_{0}^{\infty}(x, y)\right|
\end{aligned}
$$

By (5.3), the first and the last terms are bounded by $\varepsilon$. Finally, the middle term in (5.4) tends to zero as $\lambda \rightarrow 0$, by Theorem 1.3, and Theorem 5.1 follows.

\section{INITIAL VAlues that ARE MEASURES}

In this section we consider the case of initial data that may be measures. Consider first the case where the initial distribution is

$$
n_{0}^{(\lambda)}(x, y)=\sum_{m, k} \lambda_{m k} \delta\left(x-x_{m}\right) \delta\left(y-y_{k}\right)
$$

where $x_{m}$ and $y_{k}$ are all positive, $\left(x_{m}, y_{k}\right)$ belong to a finite set $A \subset P$ and $\lambda_{m k}$ are nonnegative constants, $\lambda_{m k}=0$ if $y_{k}>1$. We may try to find a solution of the form

$$
n^{(\lambda)}(x, y, t)=\sum_{m, k} R_{m k}(t) \delta\left(x-x_{m}\right) \delta\left(y-y_{k}\right),
$$


where $\left(x_{m}, y_{k}\right) \in \tilde{A} \subset P$ and $\tilde{A}$ is the closure of $A$ under impact, which is also a finite set. The $R_{m k}(t)$ satisfy the system of ordinary differential equations

$$
\begin{aligned}
\frac{d R_{m k}(t)}{d t}= & -\sum_{m^{\prime}, k^{\prime}} \varphi\left(x_{m}, x_{m^{\prime}}\right)\left(1-y_{k^{\prime}}\right)^{*}\left(1-y_{k}\right)^{*} R_{m^{\prime} k^{\prime}}(t) R_{m k}(t) \\
& +\frac{1}{2} \sum_{\substack{x_{i}+x_{j}=x_{m} \\
x_{i}^{2 / 3} y_{l}+x_{j}^{2 / 3} y_{q}=x_{m}^{2 / 3} y_{k}}} \varphi\left(x_{i}, x_{j}\right)\left(1-y_{l}\right)^{*}\left(1-y_{q}\right)^{*} R_{i l}(t) R_{j q}(t)
\end{aligned}
$$

where

$$
R_{m k}(0)=\lambda_{m k} \text {. }
$$

One can show (as in the proof of Theorem 1.1) that the system (6.3), (6.4) has a unique solution; actually, as will be shown later on, (6.2) is the only measure-valued solution of (1.1), (6.1).

Suppose now that $n_{0}(x, y)$ is a function satisfying (1.6) and approximate it by a sequence $n_{0}^{\left(\lambda_{j}\right)}$ of measures of the form (6.1),

$$
\left\|n_{0}^{\left(\lambda_{j}\right)}-n_{0}\right\|+\left\|x\left(n_{0}^{\left(\lambda_{j}\right)}-n_{0}\right)\right\| \rightarrow 0 \text { as } j \rightarrow \infty,
$$

where $\|g\|$ stands for the total variation of the absolute value of a (signed) measure $g$. Our goal is to prove that $n^{\left(\lambda_{j}\right)}(x, y, \infty)$ exists, as a measure, and

$$
\left\|(1+x)\left[n^{\left(\lambda_{j}\right)}(x, y, \infty)-n(x, y, \infty)\right]\right\| \rightarrow 0 \text { if } j \rightarrow \infty .
$$

This implies that the distribution of the mass of $n(x, y, \infty)$ can be approximated by the distribution of the mass of $n^{\left(\lambda_{j}\right)}(x, y, \infty)$ and, therefore, by solving ordinary differential equations of the form (6.3), (6.4).

Let us recall some ideas from Melzak [11] that will be further developed in the sequel.

Define the brackets

$$
\begin{aligned}
{[f, g](x, y)=\frac{1}{2}\{} & \int_{0}^{1} \int_{0}^{x} \varphi(\xi, x-\xi)(1-\eta)\left(1-\frac{y x^{2 / 3}-\eta \xi^{2 / 3}}{(x-\xi)^{2 / 3}}\right)^{*} \\
& \times f(\xi, \eta) g\left(x-\xi, \frac{y x^{2 / 3}-\eta \xi^{2 / 3}}{(x-\xi)^{2 / 3}}\right) \frac{x^{2 / 3}}{(x-\xi)^{2 / 3}} d \xi d \eta \\
& -f(x, y) \int_{0}^{1} \int_{0}^{\infty} \varphi(x, \xi)(1-\eta)(1-y) g(\xi, \eta) d \xi d \eta \\
& \left.-g(x, y) \int_{0}^{1} \int_{0}^{\infty} \varphi(x, \xi)(1-\eta)(1-y) f(\xi, \eta) d \xi d \eta\right\}
\end{aligned}
$$

so that equation (1.1) can be written in the form

$$
\frac{\partial n}{\partial t}=[n, n] \text {. }
$$

Proceeding analogously to [11], a function

$$
n(x, y, t)=\sum_{k=0}^{\infty} a_{k}(x, y) t^{k}
$$


will be a formal solution to (1.1), (1.4) if and only if

$$
a_{0}=n_{0}, \quad a_{k+1}=\frac{1}{k+1} \sum_{i+j=k}\left[a_{i}, a_{j}\right] .
$$

The estimate

$$
\iint_{P}|[f, g]| \leq C\left(\iint_{P}|f|\right)\left(\iint_{P}|g|\right)
$$

can be established as in [11] and it can then be used to derive the inequalities

$$
\iint\left|a_{k}\right| \leq\left(\iint\left|a_{0}\right|\right) m^{k}
$$

for some positive constant $m$ depending only on $\iint\left|n_{0}\right|$. If $\iint\left|n_{0}\right|<\infty$, this yields a nonnegative solution of (1.1), (1.4) for times $t<1 / \mathrm{m}$. Since $\iint|n|=\iint n$ is nonincreasing, the solution can be continued step-by-step to all times.

We wish to extend this procedure to the case in which $n_{0}$ is a finite measure. To do this, we use the fact that the space of finite signed measures on $P$ is the dual space of $C^{0}(P) \equiv\{\psi \in C(P): \psi$ vanishes at $\infty\}$. We first notice that if $f, g \in L^{1}(P)$ and $\psi \in C^{0}(P)$ we have

$$
\begin{aligned}
\langle[f, g], \psi\rangle \equiv & \int_{0}^{1} \int_{0}^{\infty} \psi(x, y)[f, g](x, y) d x d y \\
= & \frac{1}{2}\left\{\int _ { 0 } ^ { 1 } \int _ { 0 } ^ { \infty } f ( x , y ) d x d y \left(\int_{0}^{1} \int_{0}^{\infty} \chi_{0 \leq \eta \leq \frac{(x+\xi)^{2 / 3}-y x^{2 / 3}}{\xi^{2 / 3}} \varphi(x, \xi)}\right.\right. \\
& \left.\times(1-y)(1-\eta) \psi\left(x+\xi, \frac{\eta \xi^{2 / 3}+y x^{2 / 3}}{(x+\xi)^{2 / 3}}\right) g(\xi, \eta) d \xi d \eta\right) \\
& -\int_{0}^{1} \int_{0}^{\infty} f(x, y) d x d y \\
& -\int_{0}^{1} \int_{0}^{\infty} g(x, y) d x d y \\
& \left.\times\left(\psi(x, y) \int_{0}^{1} \int_{0}^{\infty} \varphi(x, \xi)(1-y)(1-\eta) f(\xi, \eta) d \xi d \eta\right)\right\}
\end{aligned}
$$

Therefore the definition of $[f, g]$ can be extended, in the obvious way, to the case in which $f$ and $g$ are finite measures. Hence, $[f, g]$ is also a finite measure with the property (cf. (6.9)).

$$
|\langle[f, g], \psi\rangle| \leq C\|f\|\|g\||\psi|_{L^{\infty}}
$$

for any $\psi \in C^{0}(P)$, and therefore

$$
\|[f, g]\| \leq C\|f\|\|g\| \text {. }
$$

Definition 6.1. A function $n: \mathbb{R}^{+} \rightarrow \mathscr{M} \equiv\{$ space of finite (signed) measures on $P\}$ is a measure-valued solution of $(1.1)$ if $(6.6)$ holds, where $[n, n]$ is defined by $(6.11)$ and $\frac{\partial n}{\partial t}$ is the strong derivative of $n$. 
Theorem 6.1. If $n_{0}$ is a nonnegative finite measure with finite $\left\|x n_{0}\right\|$, then there exists a unique measure-valued analytic solution $n(t)$ of $(1.1)$ such that $n(0)=$ $n_{0}$. The function $n(t)$ is nonnegative, and with finite $\|n(t)\|,\|x n(t)\|$ for each $t>0$.

Proof. The proof of existence was already outlined above. We seek a solution in the form (6.7) where the $a_{k}$ 's are finite measures. Then (6.6) reduces to the relations (6.8). Using the estimate (6.13), we can establish (cf. (6.10)) that

$$
\left\|a_{k}\right\| \leq\left\|n_{0}\right\| m^{k}, \quad m>0,
$$

which yields a unique analytic measure-valued solution $t \rightarrow n(t)$ for all $t<$ $1 / m$, satisfying $\|n(t)\|<\infty,\|x n(t)\|<\infty$. If we can show that $n$ is nonnegative, then $\|n(t)\|$ is nonincreasing, and proceeding step-by-step, we extend the solution to all $t$.

To prove that $n$ is nonnegative-valued we approximate $n_{0}$ by smooth nonnegative data $n_{0 j}$. The corresponding solutions $n_{j}$ are nonnegative functions (by Theorem 1.1). If we can show that

$$
\left\|n_{j}(t)-n(t)\right\| \rightarrow 0 \text { as } j \rightarrow \infty,
$$

then the nonnegativity of $n$ follows.

The assertion (6.15) is a special case of the following lemma.

Lemma 6.2. Let $n_{0}^{\sigma}(0 \leq \sigma \leq 1)$ be a family of nonnegative measures such that

$$
\left\|n_{0}^{\sigma}-n_{0}\right\| \rightarrow 0 \quad \text { if } \sigma \rightarrow 0 \text {. }
$$

Then, for any $T>0$, the corresponding solutions $n^{\sigma}(t)$ satisfy:

$$
\left\|n^{\sigma}(t)-n^{0}(t)\right\| \rightarrow 0 \text { if } \sigma \rightarrow 0, \quad \text { uniformly for } 0 \leq t \leq T .
$$

Proof. One can show that

$$
\begin{aligned}
& \text { if }\left\|a_{\sigma}-a\right\| \rightarrow 0, \quad\left\|b_{\sigma}-b\right\| \rightarrow 0 \text { as } \sigma \rightarrow 0 \\
& \text { then }\left\|\left[a_{\sigma}, b_{\sigma}\right]-[a, b]\right\| \rightarrow 0 \text { as } \sigma \rightarrow 0 .
\end{aligned}
$$

Writing $n^{\sigma}(x, y, t)$ in the form (6.7),

$$
n^{\sigma}(t)=\sum_{k=0}^{\infty} a_{k}^{\sigma} t^{k}
$$

it follows that for any $k \geq 0$,

$$
\left\|a_{k}^{\sigma}-a_{k}^{0}\right\| \rightarrow 0 \text { if } \sigma \rightarrow 0 .
$$

On the other hand (6.14) implies that

$$
\sum_{k=j}^{\infty}\left\|a_{k}^{\sigma}\right\| t^{k} \rightarrow 0 \text { if } t \leq \frac{1}{2 m}, j \rightarrow \infty
$$

uniformly in $\sigma$. Combining this remark with (6.20), the assertion (6.17) follows. 
Lemma 6.3. If $n_{0}$ is a nonnegative measure with compact support in $0 \leq x<\infty$, $0<y \leq 1$, then the corresponding solution $n_{0}(t)$ satisfies:

$$
\begin{aligned}
\left\|n_{0}(t)\right\| & =\int_{0}^{1} \int_{0}^{\infty} n_{0}(t) \rightarrow 0, \\
\left\|x n_{0}(t)\right\| & =\int_{0}^{1} \int_{0}^{\infty} x n_{0}(t) \rightarrow 0
\end{aligned}
$$

as $t \rightarrow \infty$.

Proof. We approximate $n_{0}$ by continuous nonnegative functions $n_{\sigma}(x, y)$ with support in a set $\gamma_{0} \leq y \leq 1,0 \leq x \leq x_{0}$ where $\gamma_{0}>0, x_{0}<\infty$. By Corollary 2.3, the corresponding solutions $n_{\sigma}(x, y, t)$ have, for all $t$, their support in some set

$$
\gamma_{0} \leq y \leq 2^{1 / 3}, \quad 0 \leq x \leq x_{1} \quad\left(x_{1}<\infty\right) .
$$

The inequality (3.4) holds for each $n_{\sigma}$; letting $\sigma \rightarrow 0$ and using Lemma 6.3 we deduce that (3.4) holds also for $n_{0}$. This inequality can be used to establish the relation

$$
\int_{0}^{\theta} \int_{0}^{\infty} n_{0}(t) \rightarrow 0 \text { if } t \rightarrow \infty, 0<\theta<1,
$$

as in the proof of Lemma 3.2. Here we use the fact that

$$
\iint_{S} n_{0}(t) \downarrow \quad \text { if } t \uparrow \text { provided } S \text { is impenetrable; }
$$

the proof follows by approximating $n_{0}$ with $n_{\sigma}$. Having proved (6.24) and (6.25), the proof of (6.21) follows as in Theorem 3.1. Finally, since the supports of all the $n_{\sigma}(t)$ lie in (6.23), the same is true of the support of $n_{0}(t)$. This fact together with (6.21) yields the assertion (6.22).

We now specialize to the case where $n_{0}(x, y)$ is a function (1.6) and introduce nonnegative measures $n_{0}^{\left(\lambda_{j}\right)}$ of the form (6.1) (with $y_{k}$ all positive) such that $(6.5)$ holds.

Theorem 6.4. Denote by $n^{\left(\lambda_{j}\right)}(t)$ the measure-valued solutions corresponding to $n_{0}^{\left(\lambda_{j}\right)}$. Then the limit measure $n^{\left(\lambda_{j}\right)}(\infty)$ exists and (6.5) implies that

$$
\left\|x\left(n^{\left(\lambda_{j}\right)}(\infty)-n(\infty)\right)\right\| \rightarrow 0 \quad \text { if } j \rightarrow \infty .
$$

Proof. The proof of existence of $n^{\left(\lambda_{j}\right)}(\infty)$ follows by (6.21) and monotonicity on $\{y \geq 1\}$, much as in Theorem 3.3. By Lemma 6.3, for each $j$,

$$
f_{t}(j) \equiv \int_{0}^{1} \int_{0}^{\infty} x n^{\left(\lambda_{j}\right)}(t) \downarrow 0 \quad \text { if } t \uparrow \infty .
$$

We can now proceed to establish (6.26) as in the proof of Theorem 5.1.

\section{ACKNOWLEDGMENT}

(1) We would like to thank David Ross from Eastman Kodak for suggesting the problem studied in this paper and for several useful conversations.

(2) The first author is partially supported by ARO Contract DAAL-03-88-K0110; the second author is partially supported by National Science Foundation Grant DMS-86-12880; the third author is supported by N.I.S.T. Grant No. DOC/60NANBOD1027. 


\section{REFERENCES}

1. J. M. Ball, J. Carr and O. Penrose, The Becker-Dõring cluster equation: basic properties and asymptotic properties of solutions, Comm. Math. Phys. 104 (1986), 657-692.

2. E. R. Cohen and E. U. Vaughan, Approximate solution of the equations for aerosol agglomeration, J. Colloid. and Interface Science 35 (1971), 612-623.

3. A. Friedman and F. Reitich, $A$ hyperbolic inverse problem arising in the evolution of combustion aerosol, Arch. Rational Mech. Anal. 110 (1990), 313-350.

4. E. M. Hendricks, M. H. Ernst and M. Ziff, Coagulation equations with gelation, J. Statist. Phys. 31 (1983), 519-563.

5. G. M. Hidy and J. R. Brock, The dynamics of aerocolloidal systems, Pergamon, Oxford, 1980.

6. K.W. Lee and H. Chen, Coagulation rate of polydisperse particles, Aerosol Science and Technology 3 (1984), 327-334.

7. F. Leyvraz and H. R. Tschudi, Singularities in the kinetics of coagulation processes, J. Phys. A 14 (1981), 3389-3405.

8. J. B. McLeod, On an infinite set of non-linear differential equations, Quart. J. Math. Oxford (2) 13 (1962), 119-128; (II) ibid. (2) 13 (1962), 193-205.

9. __, On a recurrence formula in differential equations, Quart. J. Math. Oxford (2) 3 (1962), 283-284.

10. __ On the scalar transport equation, Proc. London Math. Soc. (3) 14 (1964), 445-458.

11. Z. A. Melzak, A scalar transport equation, Trans. Amer. Math. Soc. 85 (1957), 547-560.

12. C. Pilinis and J. H. Seinfeld, Asymptotic solution of the aerosol general dynamic equation for small coagulation, J. Colloid. and Interface Science 115 (1987), 472-479.

13. D. Ross and T. Whitesides, A limited coalescence problem (in preparation).

14. J. L. Spouge, An existence theorem for the discrete coagulation-fragmentation equations, Math Proc. Cambridge Philos. Soc. 96 (1984), 351-357.

15. W. White, A global existence theorem for Smoluchowski's coagulation equations, Proc. Amer. Math. Soc. 80 (1980), 273-276.

(Oscar Bruno and Fernando Reitich) School of Mathematics, University of Minnesota, MinNeAPOLIS, MinNesota 55455

Current address, Oscar Bruno: School of Mathematics, Georgia Institute of Technology, Atlanta, Georgia 30332-0160

E-mail address: bruno@math.gatech.edu

Current address, Fernando Reitich: Department of Mathematics, Carnegie Mellon University, Pittsburgh, Pennsylvania 15213-3890

E-mail address: reitich@andrew.cmu.edu

(Avner Friedman) Institute for Mathematics and its Applications, University of MinNesota, Minneapolis, MinNesota 55455 